\title{
Lower layers in the motor cortex are more effective targets for penetrating microelectrodes in cortical prostheses
}

\author{
Hirak Parikh ${ }^{1}$, Timothy C Marzullo ${ }^{2}$ and Daryl R Kipke ${ }^{1,2}$ \\ ${ }^{1}$ Department of Biomedical Engineering, University of Michigan, Ann Arbor, MI, USA \\ ${ }^{2}$ Neuroscience Program, University of Michigan, Ann Arbor, MI, USA \\ E-mail: hparikh@umich.edu
}

Received 19 September 2008

Accepted for publication 3 February 2009

Published 2 March 2009

Online at stacks.iop.org/JNE/6/026004

\begin{abstract}
Improving cortical prostheses requires the development of recording neural interfaces that are efficient in terms of providing maximal control information with minimal interface complexity. While the typical approaches have targeted neurons in the motor cortex with multiple penetrating shanks, an alternative approach is to determine an efficient distribution of electrode sites within the layers of the cortex with fewer penetrating shanks. The objective of this study was to compare unit activity in the upper and lower layers of the cortex with respect to movement and direction in order to inform the design of penetrating microelectrodes. Four rats were implanted bilaterally with multi-site single-shank silicon microelectrode arrays in the neck/shoulder region of the motor cortex. We simultaneously recorded unit activity across all layers of the motor cortex while the animal was engaged in a movement direction task.

Localization of the electrode array within the different layers of the cortex was determined by histology. We denoted units from layers 2 and 3 and units as upper layer units, and units from layers 5 and 6 as lower layer units. Analysis of unit spiking activity demonstrated that both the upper and lower layers encode movement and direction information. Unit responses in either cortical layer of the cortex were not preferentially associated with contralateral or ipsilateral movement. Aggregate analysis (633 neurons) and best session analysis ( 75 neurons) indicated that units in the lower layers (layers 5,6) are more likely to encode direction information when compared to units in the upper layers (layers 2,3) $(p<0.05)$. These results suggest that electrode sites clustered in the lower layers provide access to more salient control information for cortical neuroprostheses.
\end{abstract}

(Some figures in this article are in colour only in the electronic version)

\section{Introduction}

In recent years there have been a number of advances in cortical neuroprosthetic devices and methods (Nicolelis 2001, Kennedy et al 2000, Hochberg et al 2006, Velliste et al 2008). Several areas in the brain have been shown to encode movement-related signals that potentially could be further developed for neuroprosthetic applications (Scherberger et al 2005, Hatsopoulos et al 1998, Marzullo et al 2006, Taylor et al 2002). While candidate neural sources for control signals are being actively explored, the issue of how to best access these signals using implantable microelectrode arrays needs further study. The basic requirement for a broad class of neuroprosthetic devices is the ability to record unit activity reliably for many years and the ability to record action potentials from many different cells within a small volume of cortex (Schwartz 2004). Work from various studies concludes that neuroprostheses would benefit from the addition of more channels, with higher recording quality and higher stability (Santhanam et al 2006, Patil and Turner 2008). However, practical constraints at the level of the neural interface result in cost-benefit tradeoffs. Higher site counts require more 

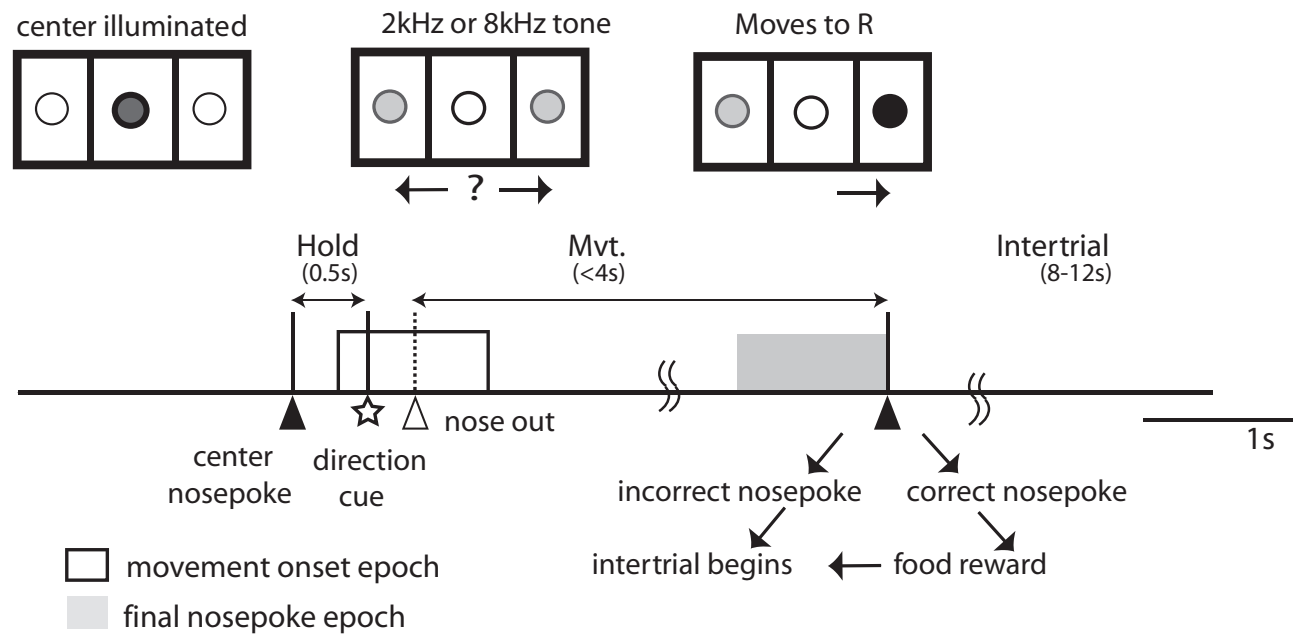

Figure 1. Behavioral paradigm: the behavioral task was a two-direction movement discrimination task. When the center nosepoke was illuminated, the animal self-initiated the task by poking the center nosepoke. After a fixed hold period of $0.5 \mathrm{~s}$, a pure tone $(2 \mathrm{kHz}$ or $8 \mathrm{kHz})$ played cueing movement to the left or right nosepoke. The animal then inserted its nose into the left or right nosepoke. If the animal failed to hold for the minimum period, the trial was aborted. If the animal correctly moved to the cued nosepoke, it was rewarded with a food pellet. The trial was ended following a correct or incorrect nosepoke. After a variable intertrial period of 8-12 s, the center nosepoke was illuminated again to indicate that the next trial could be initiated. The two boxed regions denote the two analysis epochs: the 'movement onset' epoch, which is the $1 \mathrm{~s}$ window around movement onset shown by the dashed line (variable due to reaction time delay); and the 'final nosepoke' epoch, which is the $1 \mathrm{~s}$ window before the final nosepoke.

complex packaging and multiplexing electronics. Additional penetrating shanks may increase the incidence of deleterious reactive tissue response. A top-down design approach would be to consider interface designs that are likely to yield the most salient control-related information with the fewest number of electrode sites and penetrating shanks. Anatomically, the neocortex has a regular columnar structure consisting of six layers that is similar across different brain areas (DeFelipe et al 2002). Specifically, how does the signal quality of recording in the different layers guide electrode design? How should electrode sites in the six-layered cortex be distributed to maximize salient control information?

Previous neuroprosthetic research has typically targeted neurons in the lower layers of the motor cortex (layers 5, 6) (Serruya et al 2002, Taylor et al 2003, Donoghue 2002) to obtain a control signal because the large pyramidal Betz cells in these layers project to the spinal cord, and their large dipole fields result in higher recording quality relative to other cells (Humphrey et al 1970). Advances in electrode technology and the ability to conduct long-term, simultaneous, multi-site recordings have made it possible to evaluate event-related action potentials from different cortical layers for movement and direction information. In this study, four rats were implanted bilaterally with single-shank multisite silicon microelectrode arrays and trained to perform a two-direction movement discrimination task. We used fixed microelectrodes, similar to electrodes used in long-term neuroprostheses, to compare unit activity in the upper layers $(2,3)$ and lower layers $(5,6)$ with respect to movement and direction across and within sessions. To localize the electrode sites in the different layers, we used a combination of selective microlesioning and Nissl staining of cortical slices for post mortem reconstruction of the electrode track. We found units in both the upper and lower layers encode movement and direction, but they do not appear to have an ipsilateral or contralateral direction preference. More importantly, units in lower layers of the cortex are more likely to encode directional information as compared to units in the upper layers. Our study suggests that electrodes with sites clustered in the lower layers will be more effective in obtaining control signals for neuroprosthetic applications.

\section{Methods}

\subsection{Behavioral paradigm}

Prior to surgery, animals were food deprived to $85 \%$ of their free-feeding weight and trained for $2-3$ weeks on a twodirection movement discrimination task (Cohen and Nicolelis 2004) using a three-aperture nosepoke. It should be noted that this is an all-body movement task, which is substantially different from the reaching and grasping tasks typically performed in non-human primate experiments. The behavioral paradigm is shown in figure 1 . At the start of each trial, the center nosepoke was illuminated and the rat self-initiated the trial by inserting its nose into the center nosepoke. After a fixed hold period of $0.5 \mathrm{~s}$, a pure tone of $2 \mathrm{kHz}$ or $8 \mathrm{kHz}$ (in the auditory range of the rat (Otto et al 2005)) was played which cued the rat to move to the left or right nosepoke, respectively. If the animal failed to hold for the minimum period before the tone, the trial was aborted. If the rat responded by inserting its nose in the cued nosepoke, it was rewarded with a $45 \mathrm{mg}$ food pellet (Bio-Serv, Frenchtown, NJ) and the intertrial period began; if the animal responded by inserting its nose in the non-cued nosepoke, the trial was ended and the intertrial period began. After a random intertrial period of 8-12 s, the center nosepoke was illuminated again to indicate that the next trial could be initiated. Video analysis of the task 
showed stereotypical movements of nose removal from the center nosepoke and movement of the neck/head to one of the two nosepokes. For trials in which the rats took longer than $4 \mathrm{~s}$ to respond, the rats tended to pull their nose out and walk around before inserting their nose in the left or right nosepoke. Thus, to avoid confounding the results, all trials with movement times greater than $4 \mathrm{~s}$ were excluded from analysis. There were 100-150 such trials during each session, and typically one session was run per day. At the end of the session, rats were supplemented with standard rat food pellets to keep them at $85 \%$ of their free-feeding weight. Animals were kept on a reversed $12 \mathrm{~h}$ light/dark schedule and run during the dark cycle.

\subsection{Surgical implantation and preparation}

Once the behavioral paradigm was mastered by the animal ( $>85 \%$ correct trials, typically 3 weeks), we implanted the electrode arrays. We did not note a bias toward one side during the training period for any of our rats. Error rates were similar for both sides. Four male Long-Evans rats (Charles River Labs, Boston, MA) were implanted in the neck region of the motor cortex (M1) with a single-shank 16-site siliconsubstrate microelectrode (c1x16-6mm100-1250, NeuroNexus Technologies, Ann Arbor, MI). All animals had two such implants, one in each hemisphere of the cortex with stereotaxic coordinates: A.P.+3.0, M.L. \pm 2.5 (Donoghue and Wise 1982). All arrays were $6 \mathrm{~mm}$ long, had site sizes of $1250 \mu \mathrm{m}^{2}$, and $100 \mu \mathrm{m}$ spacing between each of the sixteen electrode sites. Surgery was performed as previously described in Vetter et al (2004), Marzullo et al (2006). All surgical procedures were carried out with University for Laboratory Animal Medicine (ULAM) and University Committee on Use and Care of Animals (UCUCA) approved protocols at the University of Michigan. Anesthesia was maintained through intraperitoneal injections of a mixture of $50 \mathrm{mg} \mathrm{ml}^{-1}$ ketamine, $5 \mathrm{mg} \mathrm{ml}^{-1}$ xylazine and $1 \mathrm{mg} \mathrm{ml}^{-1}$ acepromazine at an injection volume of $0.125 \mathrm{ml} / 100 \mathrm{~g}$ body weight. At every subsequent hour of surgery, $0.1 \mathrm{ml}$ ketamine $\left(50 \mathrm{mg} \mathrm{ml}^{-1}\right)$ was delivered to the animal to maintain anesthesia. Each animal was secured to a standard stereotaxic frame, and four stainless-steel bone screws were inserted into the skull. A stainless-steel ground wire attached to the electrode connector was connected to one bone screw over the cerebellum as temporary mechanical support until the connector was permanently cemented to the skull using dental acrylic. It also later served as an electrical ground point. A craniotomy ( $2 \mathrm{~mm}$ diameter hole) was performed over the target cortical area, and the dura mater was cut away to reveal the cortical surface. The electrode array was inserted by hand using \#5 fine PTFE-coated forceps into the target cortical area. Typically, the electrode was inserted such that the top site was just below the cortical surface. The electrode assembly was wrapped with GelFoam (Pfizer, Inc., New York) and then cemented with dental acrylic. The skin around the acrylic was tightened with sutures and anti-bacterial cream was applied. Animals were given 3-4 days to recover post-surgery before experiments were resumed. As a control to verify if responses were not a result of afferent feedback, we also performed passive left and right nosepoke movements on an additional set of two anesthetized animals with identical electrode arrays and locations in the motor cortex. Our results indicated that passive driving does not affect the results from this study as there were no significant differences in unit firing rates between left and right movements.

\subsection{Recording procedure}

After recovery from the surgery, animals continued to perform the same behavioral task while neural activity was recorded from both hemispheres. Spike times, spike waveforms, local field potentials (LFPs) and external events were recorded simultaneously using a Plexon Multichannel Acquisition Processor (MAP, Plexon Inc., Dallas, TX). The signal from each electrode was passed through a high-input impedance headstage with unity gain and then filtered to extract the spike and the LFP components. For spike recordings, the signals were filtered with a passband of $154-8800 \mathrm{~Hz}$, further amplified, and sampled at $40 \mathrm{kHz}$. Thresholds were manually set and spike waveforms were stored from $150 \mu$ s before to $700 \mu$ s after threshold crossing. Events were sampled at $25 \mu \mathrm{s}$ resolution. Unit activity was sorted offline from each channel using Offline Sorter (Plexon Inc., Dallas, TX). Figure 2 shows sample waveforms, ISIs and PCA clusters from all animals. Data were recorded in a single session each day, for a period of 4-6 weeks.

\subsection{Microlesioning and histology}

At the end of the study, we performed microlesioning followed by histological analysis of Nissl-stained cortical slices to determine the electrode site locations within the different layers. First, we measured $1 \mathrm{Khz}$ site impedances, and selected three to four sites that had impedances less than $1.5 \mathrm{M} \Omega$ and were approximately at the top, middle and bottom of each electrode array. At these selected electrode sites we passed $35 \mu \mathrm{A} \mathrm{dc}$ for $2 \mathrm{~s}$ using a potentiostat (AUTOLAB, Eco Chemie, Netherlands) to create micro-lesions (these parameters were determined empirically by unpublished experiments in our lab). We waited 2-3 h for microlesion 'scars' to form, and then perfused the animal intracardially with $4 \%$ paraformaldehyde and explanted the brain tissue. In all cases, electrode arrays extracted from the brain were intact and attached to the skull/headcap. After fixing the tissue, we took $50 \mu \mathrm{m}$ coronal sections and then performed a standard cresyl-violet Nissl stain on the tissue slices. The slices were then analyzed under a microscope and images were taken to reconstruct the position of the electrode array based on the shank track, lesion marks and the known geometry of the probe. After determining the location of the electrode array, we identified the location of the boundary between the upper and lower layers (the start of layer 5) by analyzing the gray-level index values of the images using ImageJ (RSB, NIH: http://rsb.info.nih.gov/ij/). At the beginning of layer 5, the gray-level index increases abruptly due to the large pyramidal cells and the higher cell density.

For the purpose of this study, we denote units collected from layers 2 and 3 as upper layers, and units from layers 5 and 6 as lower layers. Layer 1 contains few recordable cell 

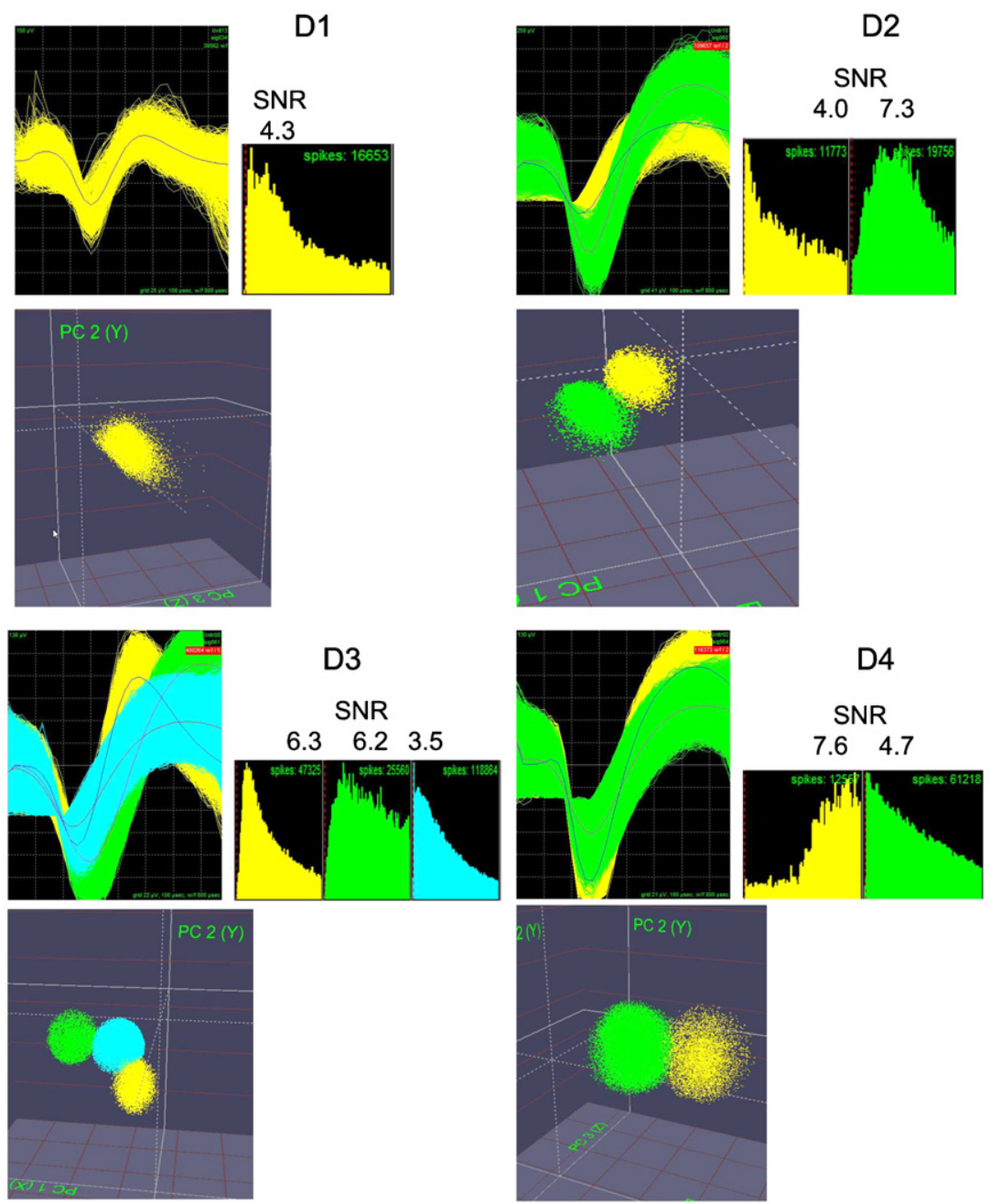

Figure 2. Sample waveforms: representative waveforms from all four animals D1-D4 showing sorted waveforms, associated SNRs, ISIs and 3D PCA clusters of sorted units.

bodies, and layer 4 is small and its existence in the rat motor cortex is debated (Brecht et al 2004). Data from the site that was closest to the determined boundary between the upper and lower layers was not used for subsequent analysis (this effectively created a $200 \mu \mathrm{m}$ separation between the upper and lower layers). This was done to account for the margin of error in determining the precise boundary between layers, leading to a possible incorrect assignment of a site at the boundary to a different layer. Figure 3 shows array tracks, electrode lesion marks and the boundary between the upper and lower cortical layers in brain slices from all animals (D1-D4), and multiple sections for one implant (D4 Left). The D1 right implant is not shown since that array was found to be non-functional after implantation.

\subsection{Sorting quality based on signal-to-noise ratio (SNR)}

Units were manually sorted based on a number of different criteria: principal component cluster analysis, auto- and crosscorrelograms, inter-spike intervals (ISIs) and wave shape. We calculated the signal-to-noise ratio (SNR) based on the individual waveforms of the different units on a channel as an additional quantitative metric. SNR was based on the individual waveforms given by the formula

$$
\mathrm{SNR}=\frac{A}{2 * \mathrm{SD}_{\text {Noise }}},
$$

where $A$ is the amplitude of the peak-to-peak voltage of the mean waveform, and $\mathrm{SD}_{\text {Noise }}$ is the standard deviation of the noise (see Suner et al (2005) for details of the method). As per the 4-point scale by Suner $e$ t al units with SNRs between 2 and 4 are considered to be 'fair'; units with SNRs $\geqslant 4$ are deemed to be 'good'. Units with SNRs below 2 were classified as 'poor' quality and were not considered for subsequent analysis.

\subsection{Measure of task-related significance}

To compare unit activity relative to the task, we computed the firing rates of the units in two analysis epochsmovement onset epoch ( $1 \mathrm{~s}$ around movement onset) and final nosepoke epoch (1 s before the final correct nosepoke and ensuing delivery of food). In the movement onset epoch, we determined units that showed a statistically significant modulation in their firing rates by comparing the $500 \mathrm{~ms}$ premovement and $500 \mathrm{~ms}$ post-movement firing rates in each trial. 


\section{Sample slices from all implants}
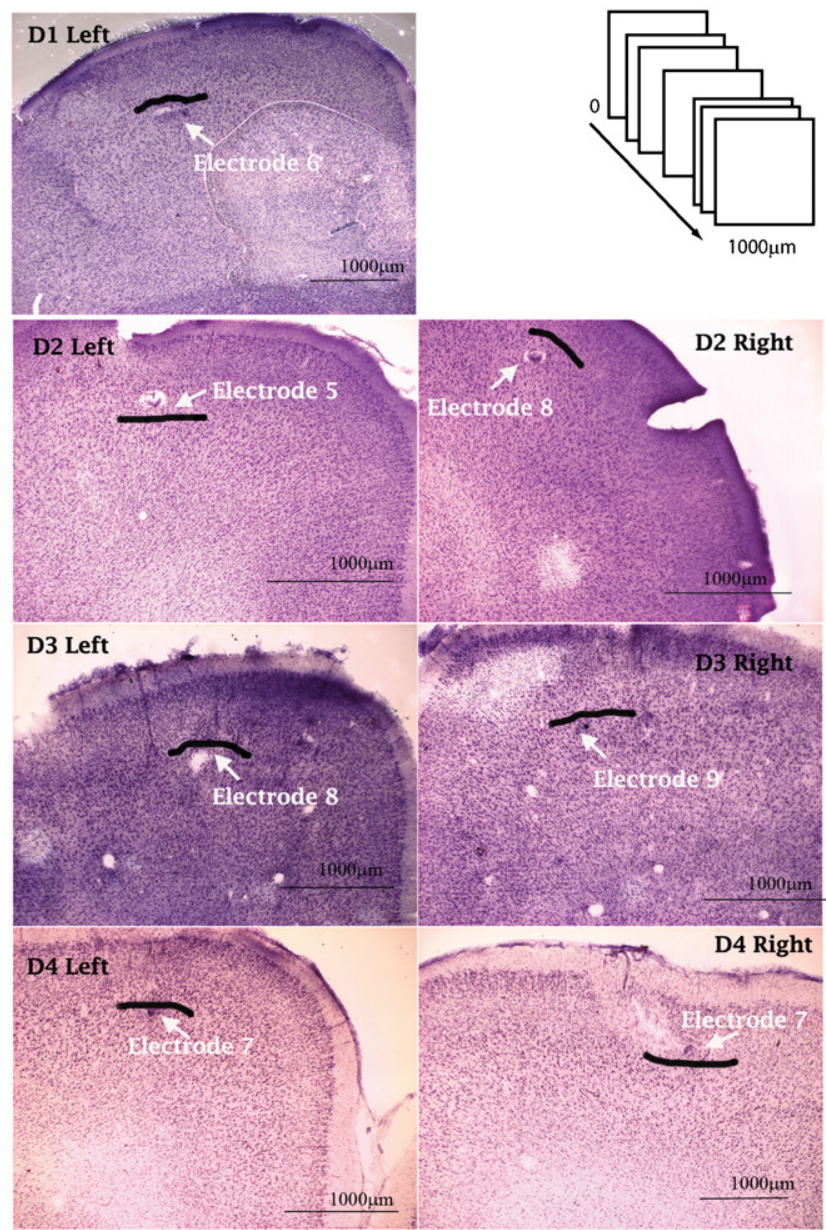

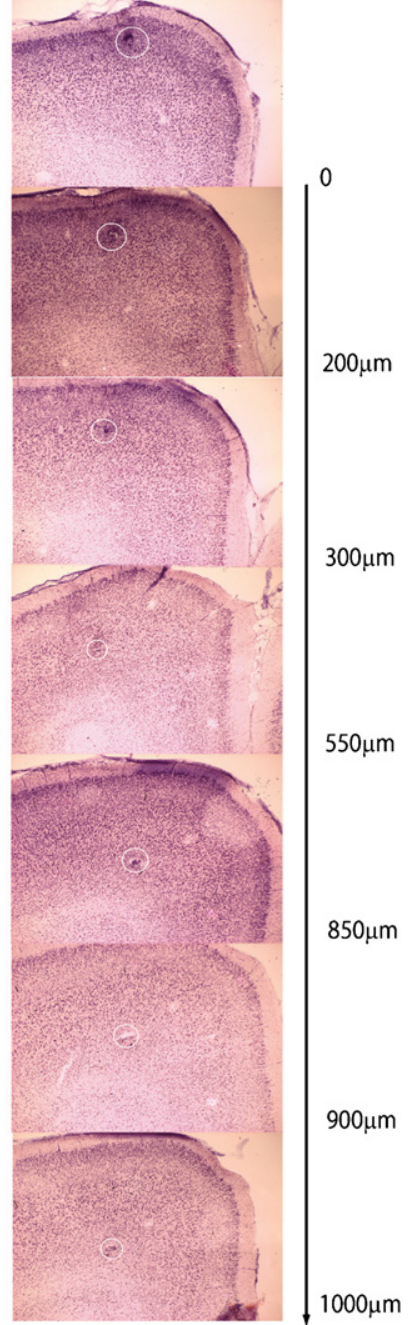

Multiple slices from D4 Left

Figure 3. Histology and electrode tracks: left panel shows Nissl-stained coronal sections of sample slices from all animals D1-D4 showing electrode tracks or lesion marks for all seven implantations. The black line marks the boundary between the upper and lower layers. The right panel shows seven coronal sections arranged rostro-caudally, as indicated by the schematic, for one implant (D4 Left) showing alternating lesions and electrode tracks which were used to reconstruct site locations.

A Mann-Whitney U-test (or Wilcoxon rank sum test) was performed to determine if there was a significant difference between the firing rates under the two different conditions for each unit (using the procedure outlined in Samejima et al (2005)). Similarly, to determine directional information we compared the unit firing rate differences between ipsilateral and contralateral movements in the entire $1 \mathrm{~s}$ movement onset epoch and $1 \mathrm{~s}$ final nosepoke epoch. Only correct trials were used for analysis. We also verified results using peristimulus time histograms (PSTHs). Spikes in the PSTHs were smoothed using a Gaussian kernel of $50 \mathrm{~ms}$ and $95 \%$ confidence intervals for the error bars were calculated using a bootstrapping procedure based on the psth routine (Chronux Project, http://www.chronux.org). While combining units from a layer for an aggregate PSTH, the individual PSTHs were normalized by the peak firing rate in the epoch under analysis. Across the 2-3 weeks of recording, unit activity at each electrode varied from day to day, presumably due to probe micromotion or due to changes in local environment as a result of probe implantation (Subbaroyan et al 2005). For aggregate analysis, we consider units from separate sessions as different units. However, this assumption is not strictly true as the same units could be present across multiple sessions. We additionally performed 'best' session analysis that only considers a single session from each electrode. For each channel, the session with the highest SNR for a unit on that channel was chosen as the 'best' session. In this analysis there are no overlapping units, as only one session per site was considered.

\subsection{Significance of difference between the upper and lower layers}

After determining which units showed task-related significance, we prepared a $2 \times 2$ contingency table layer (upper or lower) versus response (modulated, did not modulate). We sorted and summed all the units in the four categories. This analysis reveals if there is a statistically 


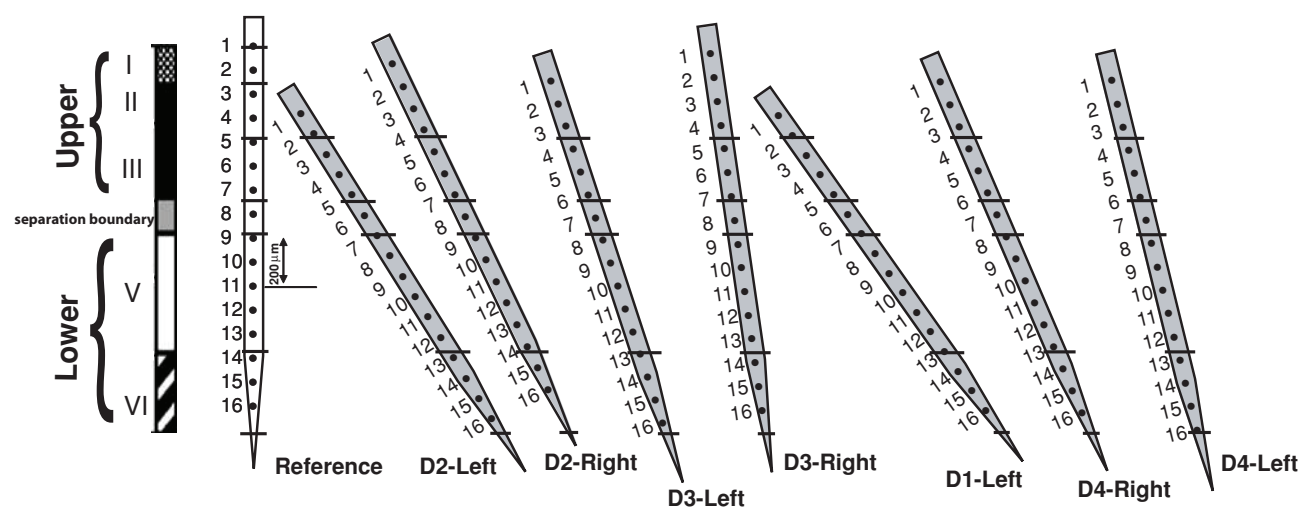

Figure 4. Implant location: cartoon shows location and orientation of the different electrode sites in the various layers for all animals (layer thicknesses are approximately scaled). The gray band is the $200 \mu \mathrm{m}$ separation region between the upper and lower layers.

significant effect of the layer location on the response characteristics of the units. For the aggregate analysis, we used a paired chi-squared $\left(\chi^{2}\right)$ test to determine if responses from the two populations were statistically significant. For the best session analysis, we used Fisher's exact test to assess significance since the sample sizes were small. All reported $p$-values are two-tailed values.

\subsection{Analysis of direction preference}

We further investigated whether units in either of the two layers had a preference for movement in the contralateral or ipsilateral direction. Each unit analyzed either encoded: no direction, contralateral direction or ipsilateral direction. We performed multinomial logistic regression using the different direction preferences as categorical variables and denoted the upper and lower layers as regressors ( 0 and 1 , respectively) to predict the probability or odds ratio of observing a particular direction. A significant regressor would indicate an ipsilateral or contralateral direction preference depending on the layer from which the neuron was recorded. We calculated the regression coefficients, odds ratios and $p$-values (mnrfit routine, MATLAB, Mathworks Inc., Natick, MA). A sample odds ratio calculation is given below

$$
\begin{gathered}
\left.\ln [\text { odds ratio }]=\beta_{0}+\beta_{1} \text { (layer }\right) \\
\left.\ln \left(\frac{p(\text { contralateral })}{1-p(\text { contralateral })}\right)=\beta_{0}+\beta_{1} \text { (layer }\right) \\
p(\text { contralateral })=\frac{e^{\beta_{0}+\beta_{1} \text { (layer) }}}{1+e^{\beta_{0}+\beta_{1} \text { (layer) }}} .
\end{gathered}
$$

Here, the odds ratio predicts the relative probability or relative odds of observing a contralateral preference with respect to an ipsilateral preference given that these observations were made in the upper or lower layer, respectively. This odds ratio is predicted by the regressors $\beta_{0}$ (effect independent of layer) and $\beta_{1}$ (contribution to effect of the layer). Thus, for significant contribution of a layer for contralateral preference, the absolute value of $\beta_{1}$ should be large and significant, and the odds ratio positive. If $\beta_{0}$ is large and significant in comparison, we can conclude that most of the contribution to the side preference is independent of the layer under consideration.

\section{Results}

\subsection{Site locations}

Figure 4 depicts a schematic of the site locations of all the electrodes in the different layers. Layer thicknesses in the schematic are as per (Hutsler et al 2005) and are approximately to scale. One array in animal D1 in the right hemisphere was found to be non-functional. All following analyses are based on seven implantations in the animals D1-D4. Site locations were found to be well distributed across all layers.

\subsection{SNR quantification}

'Aggregate' analysis pooled units from all channels and sessions. All units used for analysis had SNRs at least $\geqslant 2.0$ and no other criteria was used to exclude units recorded from analysis. For the upper layers, the SNR was mean $=4.51$, $\mathrm{sd}=1.77$ and for the lower layers the $\mathrm{SNR}$ was mean $=4.42$, $\mathrm{sd}=1.75$. The best session was determined to be the session which had the largest SNR for a particular channel across all sessions for a particular electrode. Mean SNR was 5.72 and 5.95 for the upper and lower layers, respectively. There was no statistical difference in the quality of units from the two layers in both best session and aggregate analysis. Thus, there were no recording quality differences between units recorded from the upper and lower layers that would affect subsequent results.

\subsection{Movement and direction related activity}

Only correct trials with a response time less than 4 s posttone were used for analysis. Overall behavioral performance across all the sessions used for analysis was as follows: D1: 85.4\% correct, D2: $96.5 \%$ correct, D3: $75.7 \%$ correct, D4: $86.2 \%$ correct. We investigated the performance of the layers across sessions for the fixed electrodes in the aggregate and best session analysis. The aggregate analysis considered all units recorded from all channels and sessions as independent units; we recorded from 313 units in the upper layers and 320 units in the lower layers from the seven implantations. Since units recorded in each successive session are not necessarily independent, we also performed a best session 
(a)

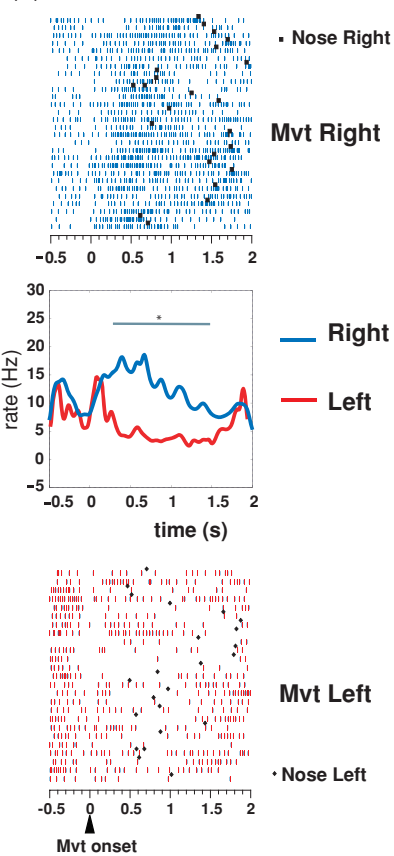

(b)

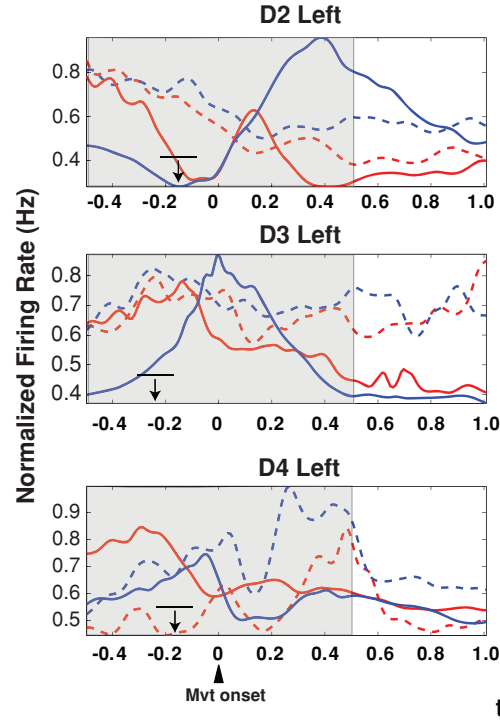

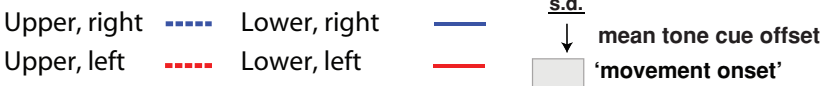
analysis epoch

D2 Right
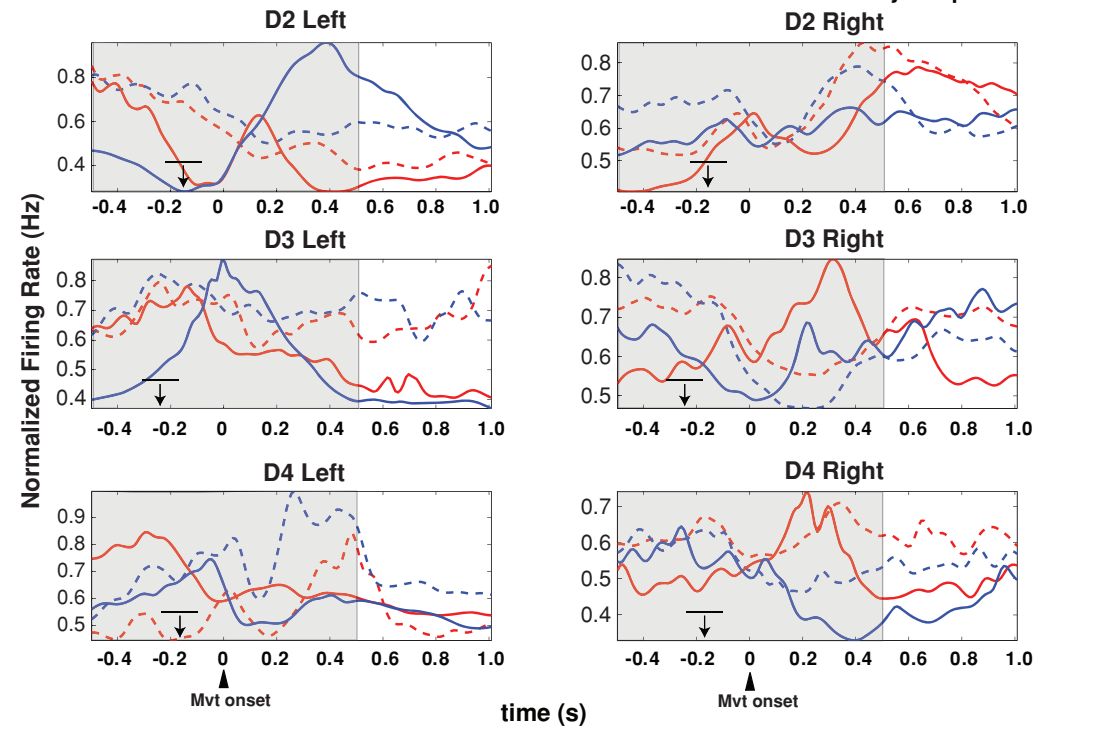
D4 Right

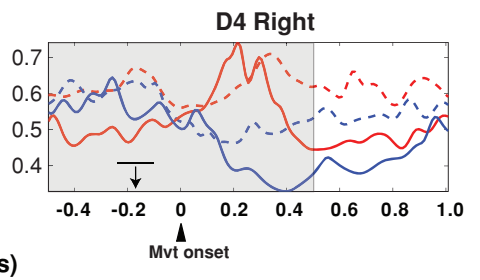

(s)

Figure 5. (a) Top and bottom panels: raster plots of a typical unit from implant D2 left for all trials separated by movement to the right and left, respectively. Dots indicate the time of the final nosepoke. Middle panel: event-triggered PSTH. Bar denotes time period where there was significant difference in firing rate between the two conditions (corrected for multiple comparisons). (b) Normalized PSTHs for units that encoded contralateral movement in the movement onset epoch. Trials were aligned to the start of movement indicated by the black triangle at $t=0$; the movement onset epoch analysis window is shown in gray. The tone cues were distributed around the mean offset indicated by the arrow, bar denotes the standard deviation.

analysis that considered units within a single session for that channel. For the best session analysis, we had 33 units from the upper layers and 42 units from the lower layers. For both sets, we analyzed unit activity in two different epochs: the movement onset epoch (1 s period around the onset of movement) and before final nosepoke ( $1 \mathrm{~s}$ period before food delivery) as shown in figure 1 to determine if encoding in the two layers changed as a consequence of behavioral state. Figure 5(a) shows sample raster plots and PSTHs for one sample unit. Figure 5(b) shows PSTHs for all animals; drop arrows and the bars correspond to the mean reaction time and associated standard deviation, respectively. We followed the non-parametric permutation approach for significance testing (Womelsdorf et al 2006) to correct for multiple comparisons in figure 5(a).

\subsubsection{Movement encoding. When we compared firing rates} of units in the movement onset epoch, both the upper and lower layers contained units that increased or decreased firing with respect to movement and direction. Figure 6 summarizes results from the aggregate and best session analysis with respect to movement encoding. We determined that there was no significant difference between units in the upper and lower layers with respect to movement in the aggregate analysis $(p=0.69)$. Units in the lower layers had a significantly greater tendency to increase firing rate with respect to movement $(p=0.04)$. We observed that 119 out of $191(62.3 \%)$ units in the lower layers showed an increase in firing as compared to 94 out of $182(51.6 \%)$ units in the upper layers. In the best session analysis we found that the difference between the upper and lower layers in terms of encoding movement was also not statistically significant $(p=0.06)$.

3.3.2. Direction encoding. Figure 7 summarizes results from aggregate and best session analysis with respect to direction encoding for both the movement onset and final nosepoke epoch. When we compared direction encoding in the aggregate analysis, the lower layer units were found to be significantly more likely to encode direction as compared to units in the upper layers in both the movement onset $(p=0.03)$ and final nosepoke epoch $(p=0.0002)$. In the best session analysis, units in the lower layers were found to be significantly more likely to encode direction of the movement as compared to units in the upper layers in the movement onset $(p=0.03)$ and final nosepoke epoch $(p=0.02)$. It has been reported that some neurons in the motor cortex respond to non-spatial sensory cues and are independent of movement direction (Salinas and Romo 1998). We compared neural activity during correct (hit) and incorrect (error) trials using a permutation test, as described in Salinas and Romo (1998), Britten et al (1996), on all the directionally tuned neurons in sessions with at least five error trials in the two error classes. We were not able to perform this analysis on any of the sessions from rat D2 since the error rates were low (incorrect trials $<4 \%$ ). Upon analysis of the data, we found that 4 out of 38 $(10.5 \%)$ neurons from the upper layers, and 4 out of $41(9.7 \%)$ in the lower layers were found to be encoding sensory rather 


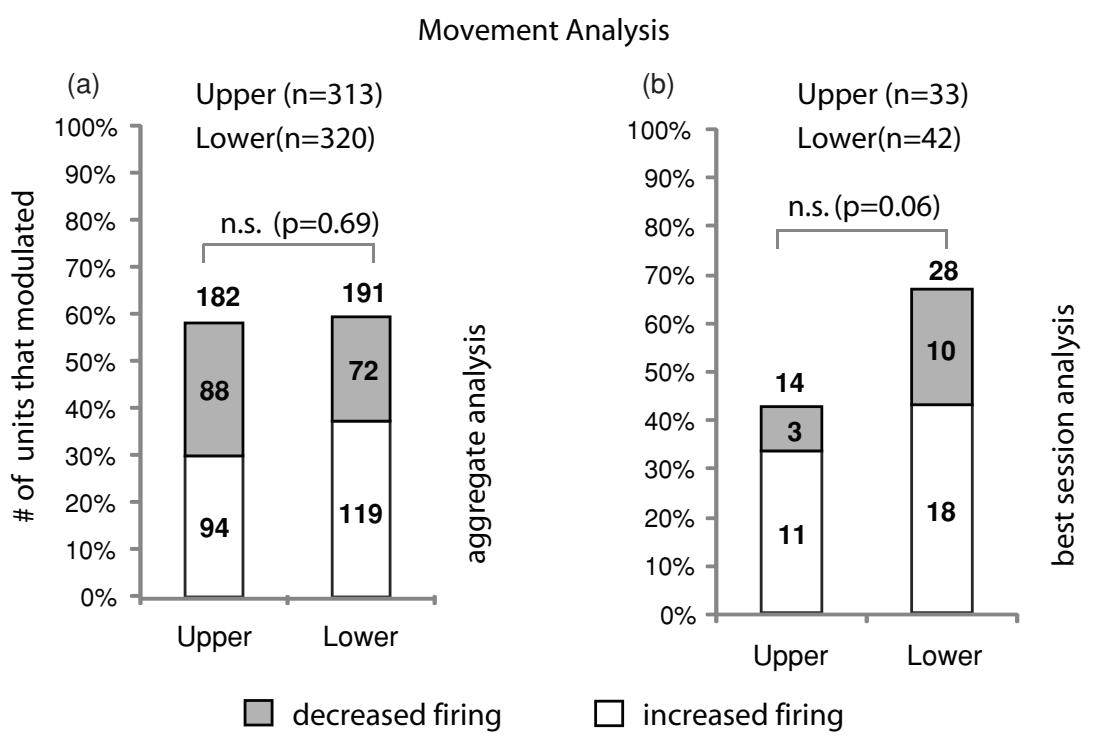

Figure 6. Movement encoding: bar graphs show the percentage of units that showed an increase or decrease in firing rate with respect to layer. (a) Aggregate analysis shows no statistically significant difference $(p=0.69)$ in the total number of units that modulated activity between the upper $(n=313)$ and lower layers $(n=320)$. Upon consideration of the kind of modulation, units in the lower layers were significantly more likely $(p=0.04)$ to increase than decrease their firing rate. We were unable to detect any such preference for the modulating units in the upper layers. (b) Best session analysis shows no statistically significant difference $(p=0.06)$ in the modulation of the unit firing rate between the upper $(n=33)$ and lower layers $(n=42)$ with respect to movement.

than motor information. After accounting for these neurons, the conclusions remain unchanged.

\subsubsection{Ipsilateral versus contralateral encoding. We further} investigated whether units in the upper or lower layers had a preference for ipsilateral versus contralateral movement. We hypothesized that the lower layers would show a contralateral preference given the large number of output neurons to the contralateral side in the lower layers of the cortex. Among the direction encoding units, we found that both layers encode ipsilateral and contralateral movements. For the log odds ratio of contralateral versus ipsilateral direction given by equation 2 , we obtained $\beta_{0}=-0.32(p=0.09)$ and $\beta_{1}=0.32$ $(p=0.20)$ for the movement onset epoch; and $\beta_{0}=-0.20$ $(p=0.27)$ and $\beta_{1}=0.32(p=0.13)$ for the final nosepoke epoch. We found units in both the upper and lower layers encode movement and direction, but do not appear to have an ipsilateral or contralateral direction preference. Data from this study suggests that both ipsilateral and contralateral direction can be obtained from a single cortical implant in one hemisphere. The lack of contralateral bias could be due to the implants being in the neck/head region which are more medially located and are expected to have more of a bilateral drive.

\section{Discussion}

\subsection{Implications for electrode and algorithm design for neuroprosthetics}

A viable long-term neuroprosthetic device requires the recording electrode arrays to be chronically implanted and held fixed. The objective of the aggregate analysis was to assess the performance of chronically implanted penetrating microelectrodes across sessions. Apart from requiring that units have an SNR above 2.0, no other selection criteria was applied to the units recorded across the different sessions. Thus, the aggregate analysis presents pooled results from different sessions under the realistic constraint that electrodes, once implanted, are not adjusted. Since the electrodes were held fixed, units across sessions for a given implant are not necessarily independent. Therefore we also investigated movement and direction performance of units in the two layers considering only a single session for each site. Best and aggregate analysis showed that units in both the upper and lower layers encode movement. About $60 \%$ of the units recorded from both layers encoded discernible movement information, and there was no statistically significant difference between them. Upper layers showed about equal number of units that increased and decreased firing with respect to movement; in contrast, units in the lower layers had a tendency to increase firing. This difference could be due to neurons in each layer being involved in different cortical microcircuits.

A simple model of cortical processing hypothesizes that the superficial pyramidal neurons combine feedforward input from subcortical, inter-areal and intra-areal excitatory sources and explore possible interpretations, whereas the deeper layers are organized to exploit the evolving interpretations of these signals (Douglas and Martin 2004). The layer 5 pyramidal cells process the local superficial signals and converge their output to motor structures. The outputs also drive subcortical structures such as the basal ganglia, red nucleus, cerebellum and ventral spinal cord. Recent research suggests this descending chain of events by demonstrating that the upper layer neurons act like preamplifiers driving output neurons in 


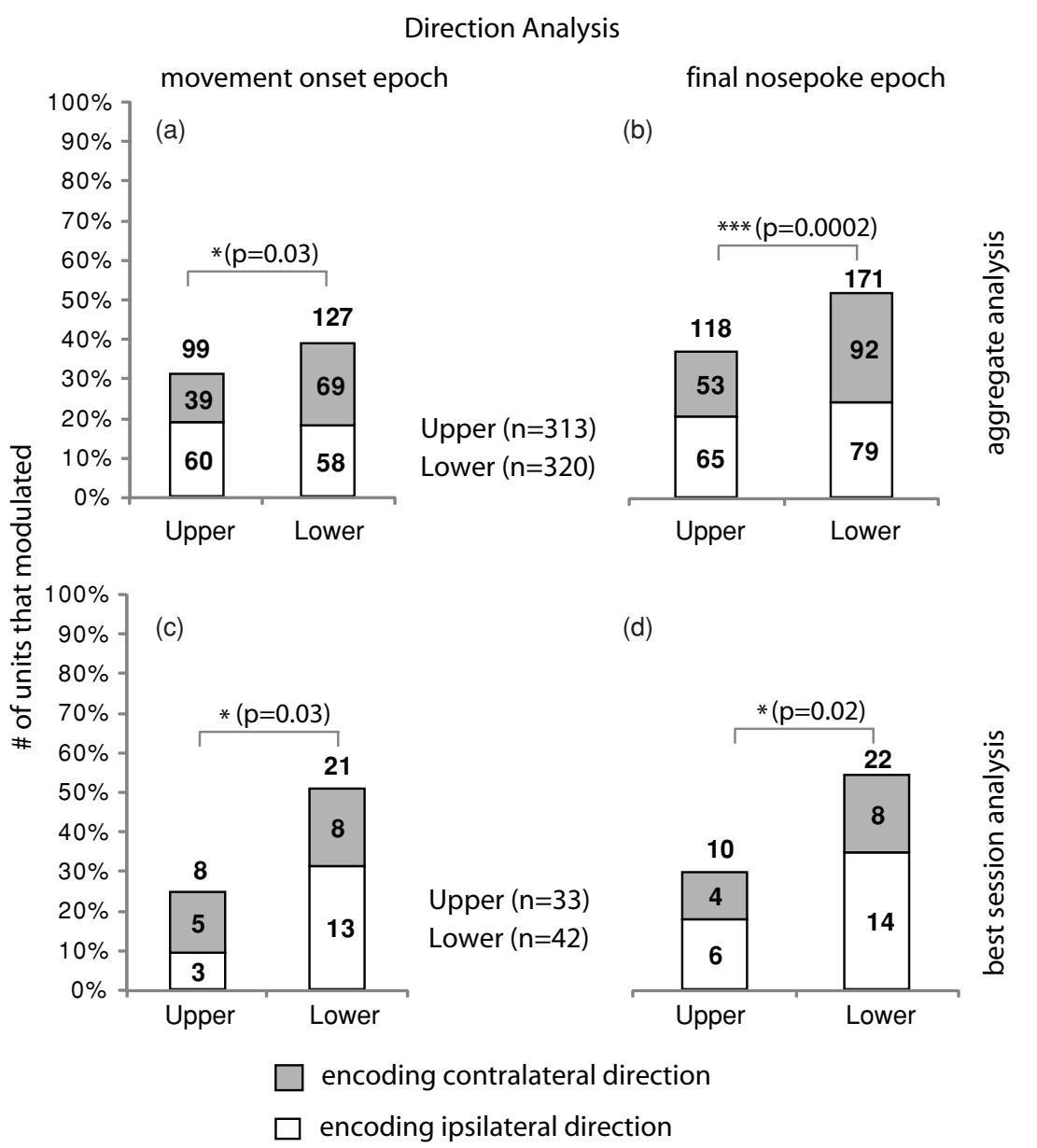

Figure 7. Direction encoding: bar graphs show the percentage of units that showed an ipsilateral or contralateral direction preference with respect to layer. (a) and (b) Aggregate analysis shows statistically significant differences in the movement onset $(p=0.03)$ and final nosepoke epochs $(p=0.0002)$, in modulation of the unit firing rate in the upper $(n=313)$ and lower layers $(n=320)$ with respect to direction encoding. (c) and (d) Best session analysis shows statistically significant differences in the movement onset $(p=0.03)$ and final nosepoke epochs $(p=0.02)$, in modulation of the unit firing rate in the upper $(n=33)$ and lower layers $(n=42)$ with respect to direction encoding.

the lower layers (Weiler et al 2008). This implies that the upper layer neurons play the role of network controllers as they inhibit and excite downstream neurons, while lower layer neurons select specific motor outputs. Our results suggest that lower layers are more salient targets for control signals as this will maximize control information per electrode site. In terms of single-shank electrodes, we suggest that sites should be clustered at the end of the shank to record from deeper layers instead of being uniformly spread along the shank. This does not necessarily require an increase in length of the probe, but an alteration of site spacing.

Several studies have found a variety of arm directions encoded by neurons recorded with a single multi-site implant in one hemisphere (Taylor et al 2002, Velliste et al 2008). In our study, when the direction preference was considered, both layers showed similar ipsilateral and contralateral tuning. Since predominant motor cortical output projects to the contralateral side of the spinal cord, we expected there would be a similar preference for the contralateral side. We found units in both the upper and lower layers do not appear to have an ipsilateral or contralateral direction preference.
This is encouraging from a neuroprosthetic point of view as it precludes the necessity of implanting microelectrode arrays in both hemispheres to obtain effective ipsilateral and contralateral control, although this study only investigated a two-directional task. In our study, the implants were in the head/neck region and hence can be expected to have more of a bilateral drive which may explain the absence of an observed contralateral bias. We did not consistently observe ipsilateral cells decreasing firing or contralateral cells increasing activity. It has been previously observed in nonhuman primates that for unimanual movements, ipsilateral cells have a tuning response similar to that for contralateral movements; for bimanual movements, ipsilateral cells have preferred directions randomly shifted (Rokni et al 2003). Thus, there may not necessarily be a decrease in firing for ipsilateral movements. This study used a two-directional whole-body movement task in the rat motor cortex. Additional experiments would need to be performed in a multidirectional, 3D task using non-human primates to verify these results and parse additional movement parameter information such 
as velocity, acceleration, joint dynamics, etc, for human neuroprosthetic use.

\subsection{Minicolumns and functional extent}

From the point of view of a neuroprosthetic system, accurate characterization of architecture of the neocortex will enable better design of probe geometries to increase throughput of signals. The six-layered structure of the neocortex is roughly similar across brain areas; only the relative thickness of the layers, number of neurons and cell type differs (DeFelipe et al 2002). Observations in the cat somatosensory cortex led Mountcastle to hypothesize the concept of a minicolumn (Mountcastle et al 1957) which extends perpendicularly across all six layers and forms a basic functional unit with similar response properties. But, properties of cells have found to vary across a single orientation column in the visual cortex (Bauer et al 1983). While histological analysis revealed that all our implantations were at an angle, the optimal angle of implantation for a penetrable microelectrode in the motor cortex is yet to be determined.

In the motor cortex, the distribution of directionally tuned cells is non-uniform and highly structured in both dimensions. Specifically, M1 cells with similar preferred directions tend to condense in a vertical dimension forming an ordered structure of minicolumns perpendicular to the surface of the neocortex of width $\sim 30 \mu \mathrm{m}$ and repeating at a lateral distance of $200 \mu \mathrm{m}$ (Amirikian and Georgopoulos 2003, Georgopoulos et al 2007). According to the Amirikian et al study, in the monkey motor cortex the functional extent depthwise was $500 \mu \mathrm{m}$ (dorsal-ventral) compared to the cortical column length of $2200 \mu \mathrm{m}$; whereas in our study, we observed that the functional extent spans almost the entire vertical length of rat motor cortex as units from layers 2-6 are involved in encoding movement and direction. This difference in functional extent could be due to inter-species difference, since the number of neurons contained in a vertical cylinder of cortical tissue varies across species (DeFelipe et al 2002). In the Amirikian et al study, the sites were not well localized in the vertical direction and could have contributed to the effect of seeing a smaller resultant functional distance. Recent work by the same group has demonstrated a novel method to determine electrode locations using fluorescent dyes and registering Nissl-stained slices to investigate the organization of preferred directions in the motor cortex (Naselaris et al 2005, Naselaris et al 2006). Using this technique they recently suggested that two kinds of pyramidal cells exist in M1, and interneurons dynamically alter the preferred directions of one class of pyramidal cells which are present across layers of the cortex affecting directional information processing during the preparation and execution of reaching movements (Merchant et al 2008). These advances in techniques to determine the location and type of cell recorded in an awake, behaving preparation via extracellular recordings will help validate proposed cortical microcircuits and the functional role of the different cells across the various layers (Du et al 2008).

\section{Conclusion}

Units in both the upper and lower layers of the rat motor cortex encode movement and direction information. Analysis across sessions and within sessions showed that units in the lower layers are significantly more likely to encode direction information as compared to units in the upper layers. These results suggest that electrode geometries with sites clustered in the lower layers will provide access to more salient control information. Improved cell and layer labeling techniques, and more degrees of freedom in a behavioral task will enable us to further parse details of movement and direction encoding in the motor cortex. These results encourage further investigation into utilizing layer-specific differences in the context of a human neuroprosthesis.

\section{Author contributions}

HP ran the training and recording paradigms, performed the analysis, and was responsible for the writing of the manuscript. TCM performed all the surgeries, helped in the design of the experiment, and in the writing of the manuscript. DRK supervised the overall aspects of the study, design of the experiment, methods and the writing of the manuscript.

\section{Acknowledgments}

Preliminary results from this study were published in the Conference Proceedings of the IEEE-EMBS Society, Lyon, France. The authors would like to thank members of the Neural Engineering Laboratory (NEL) for their comments and suggestions, CSCAR (Center for Statistical Consultation and Research), Gregory Gage for the behavioral programming and suggestions, and Drs Mark Lehmkuhle and JD Berke for their advice on specific aspects of this study. This research was supported by NIH Cortical Control R21 grant from the National Institutes of Health (5 R21-HD04894202) and the Center for Neural Communication Technology, a P41 Resource Center funded by the National Institute of Biomedical Imaging and Bioengineering (NIBIB, P41 EB002030) and supported by the National Institutes of Health (NIH).

Disclosure. DRK is the founder and CEO of NeuroNexus Technologies, Ann Arbor, MI.

\section{References}

Amirikian B and Georgopoulos A P 2003 Modular organization of directionally tuned cells in the motor cortex: is there a short-range order? Proc. Natl. Acad. Sci. USA 100 12474-9

Bauer R, Dow B M, Snyder A Z and Vautin R 1983 Orientation shift between upper and lower layers in monkey visual cortex Exp. Brain. Res. 50 133-45

Brecht M, Krauss A, Muhammad S, Sinai-Esfahani L, Bellanca S and Margrie T W 2004 Organization of rat vibrissa motor cortex and adjacent areas according to cytoarchitectonics, microstimulation, and intracellular stimulation of identified cells J. Comp. Neurol. 479 360-73 
Britten K H, Newsome W T, Shadlen M N, Celebrini S and Movshon J A 1996 A relationship between behavioral choice and the visual responses of neurons in macaque MT Vis. Neurosci. 13 87-100

Cohen D and Nicolelis M A L 2004 Reduction of single-neuron firing uncertainty by cortical ensembles during motor skill learning J. Neurosci. 24 3574-82

DeFelipe J, Alonso-Nanclares L and Arellano J I 2002 Microstructure of the neocortex: comparative aspects J. Neurocytol. 31 299-316

Donoghue J P 2002 Connecting cortex to machines: recent advances in brain interfaces Nat. Neurosci. 5 (Suppl.) 1085-8

Donoghue J P and Wise S P 1982 The motor cortex of the rat: Cytoarchitecture and microstimulation mapping J. Comp. Neurol. 212 76-88

Douglas R J and Martin K A C 2004 Neuronal circuits of the neocortex Annu. Rev. Neurosci. 27 419-51

Du J, Riedel-Kruse I H, Nawroth J C, Roukes M L, Laurent G J and Masmanidis S C 2008 High-resolution three-dimensional extracellular recording of neuronal activity with microfabricated electrode arrays $J$. Neurophysiol. at press doi:10.1152/jn.90992.2008

Georgopoulos A P, Merchant H, Naselaris T and Amirikian B 2007 Mapping of the preferred direction in the motor cortex Proc. Natl. Acad. Sci. USA 104 11068-72

Hatsopoulos N G, Ojakangas C L, Paninski L and Donoghue J P 1998 Information about movement direction obtained from synchronous activity of motor cortical neurons Proc. Natl. Acad. Sci. USA 95 15706-11

Hochberg L R, Serruya M D, Friehs G M, Mukand J A, Saleh M, Caplan A H, Branner A, Chen D, Penn R D and Donoghue J P 2006 Neuronal ensemble control of prosthetic devices by a human with tetraplegia Nature 442 164-71

Humphrey D R, Schmidt E M and Thompson W D 1970 Predicting measures of motor performance from multiple cortical spike trains Science 170 758-62

Hutsler J J, Lee D-G and Porter K K 2005 Comparative analysis of cortical layering and supragranular layer enlargement in rodent carnivore and primate species Brain Res. 1052 71-81

Kennedy P R, Bakay R A, Moore M M, Adams K and Goldwaithe J 2000 Direct control of a computer from the human central nervous system. IEEE Trans. Rehabil. Eng. 8 198-202

Marzullo T C, Miller C R and Kipke D R 2006 Suitability of the cingulate cortex for neural control IEEE Trans. Neural. Syst. Rehabil. Eng. 14 401-9

Merchant H, Naselaris T and Georgopoulos A P 2008 Dynamic sculpting of directional tuning in the primate motor cortex during three-dimensional reaching $J$. Neurosci. 28 9164-72

Mountcastle V B, Davies P W and Berman A L 1957 Response properties of neurons of cat's somatic sensory cortex to peripheral stimuli J. Neurophysiol. 20 374-407

Naselaris T, Merchant H, Amirikian B and Georgopoulos A P 2005 Spatial reconstruction of trajectories of an array of recording microelectrodes J. Neurophysiol. 93 2318-30
Naselaris T, Merchant H, Amirikian B and Georgopoulos A P 2006 Large-scale organization of preferred directions in the motor cortex: I. Motor cortical hyperacuity for forward reaching J. Neurophysiol. 96 3231-6

Nicolelis M A 2001 Actions from thoughts Nature 409 403-7

Otto K J, Rousche P J and Kipke D R 2005 Cortical microstimulation in auditory cortex of rat elicits best-frequency dependent behaviors J. Neural. Eng. 2 42-51

Patil P G and Turner D A 2008 The development of brain-machine interface neuroprosthetic devices Neurotherapeutics 5 137-46

Rokni U, Steinberg O, Vaadia E and Sompolinsky H 2003 Cortical representation of bimanual movements J. Neurosci. 23 11577-86

Salinas E and Romo R 1998 Conversion of sensory signals into motor commands in primary motor cortex $\mathrm{J}$. Neurosci. 18 499-511

Samejima K, Ueda Y, Doya K and Kimura M 2005 Representation of action-specific reward values in the striatum Science 310 1337-40

Santhanam G, Ryu S I, Yu B M, Afshar A and Shenoy K V 2006 A high-performance brain-computer interface Nature 442 195-8

Scherberger H, Jarvis M R and Ra A 2005 Cortical local field potential encodes movement intentions in the posterior parietal cortex Neuron 46 347-54

Schwartz A B 2004 Cortical neural prosthetics Annu. Rev. Neurosci. 27 487-507

Serruya M D, Hatsopoulos N G, Paninski L, Fellows M R and Donoghue J P 2002 Instant neural control of a movement signal Nature 416 141-2

Subbaroyan J, Martin D C and Kipke D R 2005 A finite-element model of the mechanical effects of implantable microelectrodes in the cerebral cortex J. Neural. Eng. 2 103-13

Suner S, Fellows M R, Vargas-Irwin C, Nakata G K and Donoghue J P 2005 Reliability of signals from a chronically implanted, silicon-based electrode array in non-human primate primary motor cortex IEEE Trans. Neural. Syst. Rehabil. Eng. 13 524-41

Taylor D M, Tillery S I H and Schwartz A B 2002 Direct cortical control of 3D neuroprosthetic devices Science 296 1829-32

Taylor D M, Tillery S I H and Schwartz A B 2003 Information conveyed through brain-control: cursor versus robot IEEE Trans. Neural. Syst. Rehabil. Eng. 11 195-9

Velliste M, Perel S, Spalding M C, Whitford A S and Schwartz A B 2008 Cortical control of a prosthetic arm for self-feeding Nature 453 1098-101

Vetter R J, Williams J C, Hetke J F, Nunamaker E A and Kipke D R 2004 Chronic neural recording using silicon-substrate microelectrode arrays implanted in cerebral cortex IEEE Trans. Biomed. Eng. 51 896-904

Weiler N, Wood L, Yu J, Solla S A and Shepherd G M G 2008 Top-down laminar organization of the excitatory network in motor cortex Nat. Neurosci. 11 360-6

Womelsdorf T, Fries P, Mitra P P and Desimone R 2006 Gamma-band synchronization in visual cortex predicts speed of change detection Nature 439 733-6 